\title{
Um caso especial de integrais binômias $\left({ }^{*}\right)$
}

\author{
IZAIAS RANGEL NOGUEIRA
}

Escola Superior de Agricultura aLuiz de Queiroz»

* Recebido para publicação em 23 de março de 1960. 


\section{CONSIDERAÇOES TEORICAS}

Muitas vêzes as integrais que envolvem radicais podem ser transformadas na integral binômia da forma

$$
\int x^{\prime \prime}\left(a+b x^{4}\right)^{r} d x
$$

em que $a$ e $b$ são números reais quaisquer e $m, n$ e $p$, números racionais relativos.

Demonstra-se que a integral binômia acima é calculável elementarmente quando fôr inteiro um dos 3 números seguintes:

$$
r, \frac{p+1}{q}+\frac{p+1}{q}+r .
$$

Veja-se, por exemplo, CATUNDA (1952).

Provou-se que nāo sendo satisfeitas as condições acima a integral não pode ser obtida por meios elementares (POTRON, 1935).

No entanto, quando se tem uma soma de duas integrais binômias insolúveis, de acôrdo com o critério acima, pode-se obter em alguns casos especiais solução em forma de expressões elementares.

Isto acontecerá, por exemplo, num caso em que, decompondo pelo método de integração por partes uma das integrais binômias, obtém-se uma integral igual à que não foi decomposta, com sinal contrário desta.

Por exemplo, se

$$
A \int x^{p}\left(a+b x^{\prime \prime}\right)^{r} d x+B \int x^{p^{\prime}}\left(a+b x^{u^{\prime}}\right)^{r} d x
$$

é a soma de duas integrais binômias, A e B sendo coeficientes constantes, a decomposição da primeira por partes nos dá:

$$
\begin{gathered}
A \frac{x^{p+1}}{p+1}\left(a+b x^{4}\right)^{r}-\frac{A b r q}{p+1} \int x^{p+1}\left(a+b x^{4}\right)^{r-1} d x+ \\
+B \int x^{p^{\prime}}\left(a+b x q^{\prime}\right)^{r^{\prime}} d x
\end{gathered}
$$
será:

Se as duas integrais finais desta soma se anularem, a solução

$$
A \frac{x^{1+1}}{p+1}(a+b x q)^{r}
$$




\section{EXEMPLO NUMÉRICO}

Resolver a integral

$$
\int \frac{x-3}{\sqrt{x^{3} \sqrt{(x-1)^{4}}}} d x
$$

A fim de simplificar o radical ${ }^{s} V(x-1)^{4}$ e obter a decomposição na forma de duas integrais binômias, ponhamos:

$$
\mathrm{x}-3=\mathrm{A}(\mathrm{x}-1)+\mathrm{B} \mathrm{x}, \bullet \mathrm{A}=3, \mathrm{~B}=-2
$$

Fica :

$$
\begin{aligned}
& \int \frac{x-3}{6 \sqrt{x^{3}} \sqrt{(x-1)^{4}}} d x=\int \frac{3(x-1)-2 x}{6 \sqrt{x^{3}} \sqrt{(x-1)^{4}}} d x \\
& =\frac{3}{6} \int \frac{(x-1) d x}{\sqrt{x^{3} V(x-1)^{4}}}-\frac{2}{6} \int \frac{x d x}{\sqrt{x^{3}} \vee(x-1)^{4}} \\
& =\frac{1}{2} \int x^{-1 / 2}(x-1)^{-1 / 3} d x-\frac{1}{3} \int x^{1 / 2}(x-1)^{4 / 3} d x
\end{aligned}
$$

Verifica-se imediatamente que nenhuma das duas integrais acima pode ser expressa por funções elementares, pois $r,(p+1) / q$ $\mathrm{e}(\mathrm{p}+1) / \mathrm{q}+\mathrm{r}$ em nenhuma delas é número inteiro.

No entanto, decompondo a segunda integral, por partes, com $u=x^{1 / 2}$ e dv $=(x-1)^{-4 / 3}$, fica

$$
\begin{aligned}
& \int \frac{(x-3) d x}{6 \sqrt{x^{3}} \sqrt{(x-1)^{4}}}=1 / 2 \int x^{-1 / 2}(x-1)^{-/ 3} d x+ \\
& +x^{1 / 2}(x-1)^{-1 / 3}-1 / 2 \int x^{-1 / 2}(x-1)^{-1 / 3} d x
\end{aligned}
$$

Logo

$$
\int \frac{x-3}{6 \sqrt{x^{3} \sqrt{(x-1)^{4}}}} d x=x^{1 / 2}(x-1)^{-1 / 3}+C .
$$




\section{SUMMARY}

The present paper shows that the sum of two binomial integrals, such as

$$
A \int x^{p}\left(a+b x^{4}\right)^{r} d x+B \int x^{p^{\prime}}\left(a+b x^{u^{\prime}}\right)^{r^{\prime}} d x \text {, }
$$

where $\mathrm{A}$ and $\mathrm{B}$ are real constants and $p, q, r$ are rational numbers, can, in special cases, lead to elementary integrals. even if each by itself is not elementary. An example of the case considered is given by the integral

$$
\begin{aligned}
\int \frac{x-3}{6 \sqrt{ } x^{3} \sqrt{(x-1)^{4}}} d x & =\frac{1}{2} \int x^{-1 / 2}(x-1)^{-1 / 3} d x- \\
& -\frac{1}{3} \int x^{-1 / 2}(x-1)^{-4 / 3} d x
\end{aligned}
$$

On the rigth hand side of the last equality both integral are not elementary. But the use of integration by parts of one of them leads to the solution:

$$
\int \frac{x-3}{6 \sqrt{ } x^{3} \sqrt{ }(x-1)^{4}} d x=x^{1 / 2}(x-1)^{-1 / 3}+C .
$$

\section{LITERATURA CITADA}

CATUNDA, Omar - 1953 - Curso de Análise Matemática - III Parte. Editôra Bandeirantes, S. Paulo.

POTRON, M. L' Abbé - l.' Integrale de Différentielle Binome. Gallthier - Villars, Paris. 